\title{
Estudio descriptivo de biopsias pulmonares a cielo abierto en el Hospital Universitario San Ignacio
}

\section{Descriptive study of open lung biopsies at the Hospital Universitario San Ignacio}

\author{
INGRID PÉrez, MD(1); Darío Londoño TruJILlo, MD(2); HeRnando Russi, MD(3)
}

\section{Resumen}

INTRODUCCIÓN: la biopsia pulmonar abierta es un instrumento muy valioso para acceder a la etiología de patologías pulmonares en los casos en que no se llega a un diagnóstico con otros exámenes menos invasivos. Provee un diagnóstico definitivo en casos de sospecha de neoplasias, infecciones y enfermedades intersticiales pulmonares, especialmente en el grupo de pacientes en quienes otros procedimientos menos invasivos como biopsias trasbronquiales o percutáneas guiadas por tomografía no han sido concluyentes.

MATERIALES Y MÉTODOS: se realizó un estudio observacional descriptivo retrospectivo, en el cual se incluyó a todos los pacientes adultos llevados a biopsia pulmonar abierta entre el período de enero de 2007 hasta diciembre de 2011; los datos se obtuvieron de una base de datos de registro de procedimientos quirúrgicos realizados por el grupo de Cirugía de Tórax en las fechas descritas. Se identificó el diagnóstico médico reportado en la historia clínica y se comparó con el reporte histopatológico para evaluar si existieron cambios en el diagnóstico, pronóstico o tratamiento del paciente.

RESULTADOS: 326 pacientes fueron llevados a biopsia abierta del pulmón durante el período estudiado. Los diagnósticos más comunes en la biopsia fueron cáncer pulmonar 12\%, metástasis a pulmón $8 \%$, neumonía en organización $9 \%$, granulomas $8 \%$, antracosis $7 \%$, bulas $6 \%$, infecciones pulmonares $9 \%$. En el grupo de pacientes con diagnóstico pre-quirúrgico sospechoso de malignidad (123 pacientes), en 107 (86\%) se confirmó la sospecha de neoplasia y en $16(13 \%)$ de los casos restantes se reportó patología benigna; en el grupo de pacientes con sospecha de patología infecciosa, se obtuvo el diagnóstico por biopsia en 28 (49\%) de 57 pacientes. La mortalidad fue del 8,5\%; al revisar la causa de muerte en los pacientes, la mayoría de los casos se relacionó con la patología de base del paciente: neumonía severa, empiema, falla orgánica múltiple y no atribuibles al procedimiento quirúrgico. Tras el reporte de patología de la biopsia abierta de pulmón se cambió el diagnóstico o la conducta en el $87 \%$ de los casos, lo que representa un rendimiento mucho mayor al reportado en la literatura, que llega del $18 \%$ al $65 \%$.

CONCLUSIONES: la biopsia abierta de pulmón es un procedimiento con un bajo porcentaje de complicaciones y mortalidad, con un alto rendimiento diagnóstico, hecho que lo convierte en un elemento valiosísimo para el diagnóstico de la patología pulmonar, especialmente en el grupo de enfermedades pulmonares intersticiales, sospecha de neoplasia y casos con inmunocompromiso en los que se sospecha infección oportunista.

Palabras clave: biopsia pulmonar, histología, diagnóstico.

\begin{abstract}
INTRODUCTION: open lung biopsy is a very valuable instrument for establishing the etiology of lung diseases in cases where diagnosis cannot be reached by means of less invasive tests. It provides conclusive diagnosis in cases of suspected neoplasms, infections, and interstitial lung diseases, especially in the group of patients in whom less invasive procedures such as tomography-guided transbronchial or percutaneous biopsies have not been conclusive.
\end{abstract}

MATERIALS AND METHODS: a retrospective, descriptive, observational study was carried out, which included all adult patients taken to open lung biopsy over the period from Ja-
${ }^{(1)}$ Médica Internista y Neumóloga. Cali, Colombia.

${ }^{(2)}$ Médico Internista y Neumólogo. Jefe Médico servicio de Neumología, Hospital del Valle. Cali. Colombia. Hospital Universitario San Ignacio. Profesor Titular Facultad de Medicina, Pontificia Universidad Javeriana.

${ }^{(3)}$ Médico Cirujano de tórax. Hospital Universitario San Ignacio. Profesor Facultad de Medicina, Pontificia Universidad Javeriana. Correspondencia: Darío Londoño, Carrera 7 No. 40-62, 6-Piso, Unidad de Neumología. Correo electrónico: dlondono@javeriana.edu.co Recibido: 02-04-2013. Aceptado: 01-06-2013. 
nuary 2007 to December 2011; the data was obtained from a database in which surgical procedures performed by the Thoracic Surgery group during that period are registered. The medical diagnosis reported in the clinical record was identified and compared with the histopathological report in order to assess whether there were changes in the diagnosis, prognosis, or treatment of the patient.

RESULTS: 326 patients were taken to open lung biopsy during the study period. The most common diagnoses reached by biopsy were: lung cancer $12 \%$, lung metastasis $8 \%$, organizing pneumonia $9 \%$, granulomas $8 \%$, anthracosis $7 \%$, bullae $6 \%$, and lung infections $9 \%$. In the group of patients with pre-surgical clinical suspicion of malignancy, suspected neoplasm was confirmed in 107 (86\%), and benign disease was reported in $16(13 \%)$ of the remaining cases; in the group of patients with suspected infectious disease, diagnosis was reached by biopsy in $28(49 \%)$ of 57 subjects. The mortality rate was $8,5 \%$; most fatalities were related to the subject's baseline condition: severe pneumonia, empyema, or multiple organ failure, and the surgical procedure was not to be considered accountable. After the histopathological report of the open lung biopsy specimen, the diagnosis or management was changed in $87 \%$ of the cases, which represents a yield much greater than that reported in the literature, which ranges from $18 \%$ to $65 \%$.

CONCLUSIONS: open lung biopsy is a procedure whose rate of complications and mortality is low, while its diagnostic yield is high. This makes it a very valuable element for diagnosing pulmonary diseases, especially in the group of interstitial lung diseases, suspected neoplasm, and immunocompromised cases where opportunistic infection is suspected.

Keywords: opne lung biopsy, histology diagnosis.

\section{Introducción}

La biopsia pulmonar abierta es un instrumento muy eficaz para acceder a la etiología de patologías pulmonares en los casos en los que no hay un diagnóstico con otros exámenes menos invasivos (1). Provee un diagnóstico definitivo en casos de sospecha de neoplasias, infecciones y enfermedades intersticiales pulmonares (2), especialmente en el grupo de pacientes en quienes otros procedimientos menos invasivos como biopsias trasbronquiales o percutáneas guiadas por tomografía no han sido definitivos (3).

Hay tres grandes grupos de pacientes en quienes la biopsia pulmonar tiene gran relevancia; el primero de ellos corresponde a pacientes inmuno-comprometidos con lesión pulmonar, por ejemplo pacientes con VIH, leucemia, linfomas o uso crónico de esteroides (4). Dentro de los diagnósticos diferenciales encontrados en este grupo al ser llevados a biopsia abierta se encuentran: patologías infecciosas (30\%), intersticiales $(21 \%)$, neoplasias $(13 \%)$, daño alveolar difuso (17\%), entre otros (5). El segundo grupo lo conforman sujetos con enfermedades pulmonares intersticiales donde la naturaleza heterogénea de la lesión pulmonar no permite gran rendimiento diagnóstico con estudios no invasivos (6); en este grupo de patologías se ha reportado diagnóstico en el $92 \%$

\section{¿Qué se conocía previamente del tema?}

La biopsia pulmonar abierta se ha constituido como las estrategia diagnóstica final en el paciente con enfermedad pulmonar de causa no clara. No obstante, se ha descrito que las nuevas técnicas endoscópica y la biospia guiada por TAC ha hecho que su frecuencia se disminuya.

\section{¿Qué aporta el estudio?}

Una seria retrospectiva de casos, realizada en un centro de referencia, mostró que la biopsia pulmonar abierta muestra alto rendimiento diagnóstico, especialmente en el grupo de enfermedades intersticiales, neoplasias e inmunocomprometidos.

de los casos de neumonitis por hipersensibilidad, sarcoidosis y fibrosis pulmonar idiopática. Pero hay un $15 \%$ de patologías que aún después de la biopsia abierta quedan como neumonía intersticial no clasificable $(7,8)$. El tercer grupo corresponde a sujetos en quienes se requiere estudio de nódulos pulmonares. En este grupo se reportan diagnósticos de adenocarcinoma de pulmón (35\%), escamocelular (14\%), carcinoide (11\%) entre los más frecuentes. Dentro de las metástasis a pulmón más reportadas están: cáncer de colon, seno, riñón y melanoma.

Con la evolución de las técnicas quirúrgicas, procedimientos como las biopsias pulmonares guiadas por videotoracoscopia adquieren gran valor dada la 
menor tasa de complicaciones y menor tiempo de hospitalización $(10,11)$. Es así como en algunos centros quirúrgicos este procedimiento se realiza de manera ambulatoria (12).

Dada la conocida importancia de la biopsia pulmonar en el estudio de la patología pulmonar, se decidió realizar una revisión de las biopsias pulmonares llevada a cabo durante 2007 a 2011 en el Hospital Universitario San Ignacio y así obtener información de epidemiología local respecto a este tema. Dentro de este estudio se decidió efectuar una revisión inicial de las biopsias solo en los casos en los que se consideró una enfermedad infecciosa, lo cual permitió hacer este estudio observacional descriptivo retrospectivo donde no solo se conoció el perfil epidemiológico propio, sino hacer una retroalimentación acerca del rendimiento y la costoefectividad de los procedimientos y métodos diagnósticos en nuestro medio.

\section{Material y métodos}

Se realizó un estudio observacional descriptivo retrospectivo, en el cual se incluyó a todos los pacientes adultos llevados a biopsia pulmonar abierta entre el período de enero del 2007 hasta diciembre del 2011, cuyos datos se obtuvieron de una base de datos de registro de procedimientos quirúrgicos realizados por el grupo de Cirugía de Tórax en las fechas descritas.

En quienes se había realizado una biopsia abierta, tras la inclusión en el estudio, se revisó la historia clínica electrónica utilizando el sistema de registro del Hospital Universitario San Ignacio: SAHI. En cada uno de ellos se evaluaron variables de edad, género, motivo de consulta al grupo de cirugía de Torax, antecedentes relevantes, hallazgos de la escanografía de tórax según informe de radiología, estudios de microbiología y estudios no invasivos previos a la cirugía abierta como broncoscopias o biopsias trasbronquiales o percutáneas guiadas por tomografía. También se incluyeron variables relacionadas con la complicaciones afines al procedimiento quirúrgico, además de la mortalidad y su relación con el procedimiento. Se describió el tipo de cirugía efectuada y el hallazgo intra-quirúrgico. Paralelamente, se evaluó el tiempo con tubo de tórax, en especial para determinar el impacto de la llegada de la toracoscopia a la institución.

Se identificó el diagnóstico médico reportado en la historia clínica por el cual se solicitó la biopsia quirúrgica, el cual se considero pre-quirúrgico, y se comparó con el diagnóstico post-quirúrgico tras el reporte histopatológico de la biopsia pulmonar para evaluar si existieron cambios en el diagnóstico, pronóstico o tratamiento del paciente, o si por el contrario no hubo lugar a variables en el manejo luego de conocer el resultado de la biopsia.

Las biopsias fueron analizadas para estudios de histología, coloraciones especiales en búsqueda de infecciones en los casos seleccionados por el grupo tratante, y pruebas de inmunohistoquímica, en especial para los pacientes con sospecha o diagnóstico de cáncer.

Se utilizó estadística descriptiva para el análisis de los datos y se decidió separar las biopsias en cuatro grupos grandes: cáncer, infecciosas, intersticiales $\mathrm{y}$ varios.

\section{Resultados}

En total, se llevaron a biopsia abierta del pulmón 326 pacientes durante el período estudiado. Las biopsias fueron realizadas por toracostomía abierta, y en los últimos años por videotoracoscopia. Al sexo masculino pertenecía fue $197(60 \%)$ y al femenino $129(40 \%)$ (tabla 1$)$.

Los síntomas más reportados en la historia clínica fueron: tos $(31 \%)$, disnea (30\%), fiebre (12\%), control radiográfico en pacientes con patología tumoral (22\%), hemoptisis (2\%) y otros (1\%) (tabla 2).

En los antecedentes no fue posible determinar la historia ocupacional ni de tabaquismo en todos los pacientes; en los antecedentes patológicos se encontraron tres pacientes con diagnóstico de cáncer de pulmón y 58 (17\%) con neoplasias extra-pulmonares. Se observaron neoplasias hematológicas en 27 (8\%) pacientes y patología obstructiva pulmonar en 14 (4\%). En el grupo de pacientes con compromiso de la inmunidad el 10\% tenía diagnóstico de VIHSIDA y el 5\% usaba esteroides de forma crónica (por enfermedad reumatológica). 
Tabla 1. Distribución de las biopsias por género y por tipo de entidad.

\begin{tabular}{|l|c|c|c|c|c|}
\hline & Infección & Cáncer & Varios & Intersticiales & Todos \\
\hline Total pacientes & 57 & 123 & 91 & 55 & 35 \\
\hline Hombres & 39 & 68 & 55 & 36 & $\mathbf{1 9 7}$ \\
\hline Mujeres & 18 & 55 & 36 & 129 \\
\hline
\end{tabular}

Tabla 2. Presentación clínica.

\begin{tabular}{|c|c|c|c|c|c|}
\hline & Infección & Cáncer & Varios & Intersticiales & Todos \\
\hline Control Rx. & 2 & 58 & 13 & 0 & 73 \\
\hline Disnea & 8 & 30 & 38 & 24 & 100 \\
\hline Estridor & 0 & 0 & 0 & 0 & 0 \\
\hline Fiebre & 22 & 2 & 9 & 7 & 40 \\
\hline Hemoptisis & 1 & 1 & 3 & 3 & 8 \\
\hline Neutropenia febril & 1 & 0 & 0 & 1 & 2 \\
\hline Pérdida de peso & 0 & 1 & 1 & 0 & 2 \\
\hline Tos & 23 & 31 & 27 & 20 & 101 \\
\hline
\end{tabular}

La manifestación radiológica principal en la tomografía de tórax fue la presencia de nódulos pulmonares (tabla 3).

Dentro de los estudios de laboratorio clínico que sirvieron para confirmar el diagnóstico etiológico en 39 pacientes se encuentran ADA (adenosin deaminasa), baciloscopias, cultivos de micobacterias y PPD en 13 pacientes y para criptococo y $P$. jirovecci en solo cinco pacientes.

Del total de pacientes con biopsia pulmonar, al $30 \%$ se les realizó previamente broncoscopia diagnóstica, de las cuales el 50\% fueron normales, el $32 \%$ con endobronquitis; dentro de los otros hallazgos se describió estenosis, masas endobronquiales y sangrado en el $18 \%$ de los casos.

La mayoría de las muestras de patología se obtuvieron de biopsias en cuña vía toracotomía abierta $(65 \%)$, toracoscopia $(10 \%)$, lobectomía en 42 pacientes $(12 \%)$ y neumonectomía en 10 pacientes (3\%). Dentro de los hallazgos intra-quirúrgicos, el más informado fue nódulos pulmonares (31\%), seguido por masas (18\%), antracosis $(8 \%)$, hepatización $(6 \%)$, bulas $(4 \%)$ y normal $(4 \%)$. Otros hallazgos descritos en $28 \%$ de los casos fueron: abscesos pulmonares, adherencias, cavernas, granulomas, áreas de necrosis.

En la historia clínica no se reportaron complicaciones secundarias a la cirugía en 159 pacientes (48\%), en 62 casos las complicaciones más reportadas fueron el neumotórax residual (19\%), seguido por las atelectasias (7\%). 26 pacientes (8\%) fueron hospitalizados en la Unidad de Cuidado Intensivo, no por complicación relacionada con la cirugía sino por la patología de base. Otras complicaciones reportadas en menor grado fueron: derrame pleural con alto gasto de líquido y reintervención quirúrgica ((7 pacientes, 2\%). El tubo de tórax estuvo durante dos días en 90 pacientes (27\%), 3 días en $50(15 \%)$ y 1 día en 37 (11\%). Solo 19 pacientes tuvieron el tubo de tórax por más de 20 días (6\%).

De los 326 pacientes, los diagnósticos más encontrados en la biopsia fueron cáncer pulmonar $(12 \%)$, metástasis a pulmón (8\%), neumonía en 
Tabla 3. Patrones radiológicos de la tomografía axial de los pacientes.

\begin{tabular}{|l|c|c|}
\hline Consolidación & 36 & $11 \%$ \\
\hline Árbol en gemación & 11 & $3 \%$ \\
\hline $\begin{array}{l}\text { Nódulos } \\
\text { centrilobulillares }\end{array}$ & 9 & $3 \%$ \\
\hline Micronódulos & 8 & $2 \%$ \\
\hline Masa & 47 & $14 \%$ \\
\hline Nódulos & 78 & $24 \%$ \\
\hline Nódulo maligno & 21 & $6 \%$ \\
\hline Nódulo tejido blando & 28 & $8 \%$ \\
\hline Vidrio & 29 & $8 \%$ \\
\hline Derrame & 14 & $4 \%$ \\
\hline Otros & 45 & $14 \%$ \\
\hline
\end{tabular}

organización $(9 \%)$, granulomas $(8 \%)$, antracosis (7\%), bulas $(6 \%)$ e infecciones pulmonares (9\%). En el grupo de pacientes con diagnóstico pre-quirúrgico sospechoso de malignidad (123 pacientes), se confirmó la sospecha de neoplasia en 107 casos $(86 \%)$ y patología benigna en los $16(13 \%)$ restantes . En cuanto a los casos de cáncer pulmonar, se observó la siguiente distribución: adenocarcinoma (18 casos), bronquioloalveolar ( 7 casos), escamocelular (8 casos), neuroendocrino ( 1 caso), carcinoide (6 casos), carcinoma ( 1 caso); en aquellos con patología tumoral extrapulmonar, se logró documentar metástasis en 26 casos; otras lesiones neoplásicas en 24 pacientes y 6 pacientes con adenocarcinoma primario no conocido.

En el grupo de patología intersticial (55 pacientes), se documentaron 27 casos con neumonía en organización, 8 con neumonía intersticial no específica, 5 con neumonía intesticial linfoide, 2 con neumonía intersticial usual, 3 con neumonía de hipersensibilidad, un de proteinosis y uno de sarcoidosis, lográndose confirmar compromiso intersticial en 47 casos $(85 \%)$.

En el grupo de patología infecciosa (57 pacientes), se logró confirmar una entidad infecciosa con la siguiente distribución: abscesos (6 casos), criptococosis (6 casos), P. jirovecci (6 casos), histoplasmosis ( 4 casos), paracoccidiodomicosis ( 1 caso) y tuberculosis
(5 casos). En total se obtuvo el diagnóstico por biopsia en 28 (49\%) de 57 pacientes. Hubo un grupo de 13 pacientes en quienes se documentaron granulomas; en éstos los estudios de microbiología permitieron corroborar el diagnóstico de tuberculosis (cultivo micobacterias, ADA, PPD, baciloscopia), obteniéndose un diagnóstico definitivo en 50 de 57 pacientes.

En el grupo varios (91 pacientes), se agruparon pacientes con pruebas no diagnósticas previas a la cirugía, sin un diagnóstico probable pre-quirúrgico, y se hallaron las siguientes entidades: antracosis (14 casos), bulas (18 casos), silicosis (11 casos), granulomas ( 7 casos), hemorragia alveolar ( 4 casos), vasculitis ( 2 casos), daño alveolar difuso (3 casos), entre otros.

Se realizó una revisión del impacto de la biopsia en la situación del paciente, bien fuera por cambio de diagnóstico y/o sus implicaciones en el tratamiento o pronóstico, y se obtuvo un cambio relacionado con diagnóstico por la biopsia en $87 \%$ de los casos; en solo 40 pacientes $(12 \%)$, no se obtuvo ningún cambio, e incluso fue negativa o con resultados muy inespecíficos (tabla 4).

La mortalidad fue del 8,5\%. Al revisar la causa de muerte, en la mayoría de los casos se halló relación con la patología de base del paciente: neumonía severa, empiema, falla orgánica múltiple y no atribuible al procedimiento quirúrgico.

\section{Discusión}

Este estudio permitió conocer las características demográficas y clínicas de los pacientes llevados a biopsia pulmonar abierta en el Hospital Universitario San Ignacio entre el período 2007 y 2011. En total se recolectaron 326 pacientes, $60 \%$ hombres y $40 \%$ mujeres. Los síntomas más reportados fueron tos (31\%) y disnea (30\%). Se contó con un grupo muy especial de pacientes que pertenecen al Centro Oncológico Javeriano, con diagnóstico oncológico establecido, quienes terminan en la consulta de cirugía de tórax tras encontrar alguna anormalidad en el control radiológico regular; estos pacientes representaron el $22 \%$ de los casos.

Como se describe en la literatura, los hallazgos en la escanografía de tórax que más se relacionaron 
Tabla 4. Impacto de las biopsias y mortalidad.

\begin{tabular}{|l|c|c|c|c|c|}
\hline & Infección & Cáncer & Varios & Intersticiales & Todos \\
\hline Diagnóstico & 45 & 106 & 45 & $\mathbf{2 4 7}$ & $\mathbf{4 0}$ \\
\hline No cambios & 3 & 4 & 29 & 0 & $\mathbf{3 9}$ \\
\hline Tratamiento & 9 & 13 & 17 & 6 & $\mathbf{2 8}$ \\
\hline Muerto & 10 & 10 & $\mathbf{2}$ & 49 & $\mathbf{2 9 8}$ \\
\hline Vivo & 47 & 113 & 89 & & \\
\hline
\end{tabular}

con la solicitud de biopsia fueron la presencia de nódulos (24\%), nódulo con características radiológicas atribuibles a malignidad (6\%) y masas (14\%). Dentro de los hallazgos sugestivos de infección en pacientes inmunocomprometidos que fueron llevados a biopsia abierta, se encontraron árbol en gemación, nódulos centrilobulillares, vidrio esmerilado y consolidación en $19 \%$ de los casos.

Los estudios preliminares como broncoscopia, biopsias tras-bronquiales o percutáneas guiadas por tomografía no aportaron en gran medida al diagnóstico, y esta fue una de las principales razones de llevar a los pacientes a biopsia abierta de pulmón. En 13 pacientes se confirmó el diagnóstico de tuberculosis gracias a los resultados del laboratorio clínico. Es de aclarar que un grupo importante de pacientes no fue llevado a otras ayudas diagnósticas del laboratorio clínico en relación con la patología pulmonar; en total 161 de 326 pacientes.

En los 326 pacientes se documentaron como diagnósticos más frecuentes relacionados con la biopsia: cáncer de pulmón (12\%), metástasis a pulmón (8\%), BOOP $(9 \%)$, granulomas $(8 \%)$, antracosis $(7 \%)$, bulas $(6 \%)$ e infecciones pulmonares $(9 \%)$. En el grupo de patología intersticial se confirmó diagnóstico intersticial en 47 casos $(85 \%)$, el diagnóstico más frecuente fue neumonía en organización. En el grupo de pacientes con sospecha de patología infecciosa se obtuvo el diagnóstico por biopsia en 28 (49\%) de 57 casos.

De 326 pacientes, se tuvo cambio relacionado con el diagnóstico por el resultado de la biopsia abierta de pulmón en $87 \%$ de los casos, que es un rendimiento mucho mayor al reportado en la literatura, del 18\% al $65 \%$ (2). Es importante aclarar que se contó un grupo importante de patología tumoral con una alta probabilidad pre-test de tener un resultado positivo. Solo en 40 pacientes $(12 \%)$, no se obtuvo ningún cambio, e incluso fue negativa, o con resultados como antracosis, fibrosis, entre otros.

En la literatura se describe una tasa de complicaciones con la biopsia abierta del pulmón de hasta $7 \%$ (8); la complicación más reportada es dolor y fuga de aire. En este estudio no se reportó en la historia clínica ninguna complicación secundaria a la cirugía en $48 \%$ de los casos. La complicación más reportada fue el neumotoráx residual (19\%), pero ninguno de los pacientes requirió cambios en el tratamiento ni hubo alteración de su condición clínica. La segunda complicación reportada fue atelectasia en 7\% de los casos. Veintiséis pacientes requirieron Unidad de Cuidado Intensivo $(8 \%)$ por complicaciones relacionadas con la patología de base.

En la literatura se describe una mortalidad menor al $1 \%$ asociada con la biopsia abierta de pulmón, mientras que en este estudio se encontró una mortalidad del $8,5 \%$. No obstante, cabe aclarar que la causa de muerte estuvo más relacionada con la patología de base del paciente (neumonía severa, empiema, falla orgánica múltiple), y no necesariamente fue atribuible al procedimiento quirúrgico.

\section{Conclusión}

La biopsia abierta de pulmón es un procedimiento con un bajo porcentaje de complicaciones y mortalidad, con un alto rendimiento diagnóstico, lo cual lo convierte en un elemento muy valioso para el diagnóstico de la patología pulmonar, en especial en el grupo de enfermedades pulmonares 
intersticiales, ante posible neoplasia y en pacientes inmunocomprometidos en quienes se sospecha infección oportunista.

\section{Conflictos de intereses}

\section{Los autores declaran no tener conflictos de interés.}

\section{Bibliografía}

1. Lewis M, McKenna M, Falk J, Chaux G. Medical management of the thoracic surgery patient. Saunders Elsevier; 2009.

2. Rivas JJ, Freixinet J, Rodríguez de Castro F y Grupo Español de Cirugía Toracoscópica Videoasistida. Estudio multicéntrico español cirugía videotoracoscópica. Arch Bronconeumol. 2002;38(2):60-3.

3. Wall CP, Gaensler EA, Carrington CB, Hayes JA. Comparison of transbronchial and open biopsies in chronic infiltrative lung diseases. Am Rev Respir Dis. 1981;123:280-5.

4. Bruce D, Cheson M, Wolfram E, et al. Value of Open - Lung Biopsy in 87 Immunocompromised patients with pulmonary infiltrades. Cancer. 1985; 55: 453 -459.

5. Robbins BE, Steiger Z, Wilson RF, et al. Diagnosis of acute diffuse pulmonary infiltrates in immunosuprressed patients by open lung biopsy of the lung. Surg Gynecol Obstet. 1992;175:8-12.

6. Chan G, Yung-Chie Lee, Chen-Tu Wu, Hsao-Hsun Hsu, Pei-Ming Huang, Yih-Leong. Surgical lung biopsy for diffuse pulmonary disease: experience of 196 patients. J Thorac Cardiovasc Surg. 2005;129:984990.

7. Zegdi R, Azorin J, Tremblay B, Destable MD, Paul S. Surgical experience videothoracoscopic lung biopsy in diffuse infiltrative lung diseases: a 5-year. Ann Thorac Surg. 1998;66:1170-1173.

8. Morell F, Reyes L, Doménech G, de Gracia J, Majó J, Ferrer J. Diagnoses and diagnostic procedures in 500 consecutive patients with clinical suspicion of interstitial lung disease. Arch Bronconeumol. 2008;44:185-91.

9. Davies B, Ghosh S, Hopkinson D, Vaughan G, Rocco G. Solitary pulmonary nodules: pathological outcome of 150 consecutively resected lesions. Interact Cardiovasc Thorac Surg. 2005;4:18-20.

10. Bensard DD, Melntyre RC, Waring BJ, Simon JS. Comparison of videothoracoscopic lung biopsy to open lung biopsy in the diagnosis of interstitial lung disease. Chest. 1993;103:765-770.

11. Ferson PF, Landreneau RJ, Dowling RD, Hazelrigg SR, Ritter P, Nunchuck $\mathrm{S}$, et al. Comparison of open versus thoracoscopic lung biopsy for diffuse infiltrative pulmonary disease. J Thorac Cardiovasc Surg. 1993;106:194-199.

12. Chang AC, et al. Diagnostic thoracoscopic lung biopsy: an outpatient experience. Ann Thorac Surg. 2002;74:1942-7. 